TAMKANG JOURNAL OF MATHEMATICS

Volume 33, Number 2, Summer 2002

\title{
ON SOME USEFUL INTEGRAL INEQUALITIES AND THEIR DISCRETE ANALOGUES
}

\author{
S. B. PACHPATTE AND B. G. PACHPATTE
}

\begin{abstract}
In this paper explicit bounds on certain integral inequalities and their discrete analogues are established. To illustrate the usefulness of one of our results, some applications are also given.
\end{abstract}

\section{Introduction}

Integral inequalities which provide explicit bounds on unknown functions and their discrete analogues are frequently used to study the different properties of the solutions of various differential, integral and difference equations, see [1-8] and the references cited therein. However, the bounds provided by the inequalities available in the literature do not apply directly in some situations and it is desirable to find bounds on certain integral inequalities and their discrete analogues, which are useful in some new applications. The main purpose of this paper is to establish explicit bounds on certain integral inequalities and their discrete analogues, which will be equally important to achieve a diversity of desired goals. Some immediate applications of one of the result to convey the importance of our results to the literature are also given.

\section{Statement of Results}

In what follows, $R$ denotes the set of real numbers, $R_{+}=[0, \infty), N_{0}=\{0,1,2, \ldots\}$ are the given subsets of $R$ and $/$ denotes the derivative. The partial derivatives of a function $z(x, y), x, y \in R$ with respect to $x$ and $y$ are denoted by $D_{1} z(x, y)$ and $D_{2} z(x, y)$ respectively. We assume that all the functions which appear in the inequalities are realvalued and use the usual conventions that empty sums and products are taken to be 0 and 1 respectively.

For $t, s, \sigma \in R_{+}, 0 \leq \sigma \leq s$ and some real-valued nonnegative continuous functions $p(s), q(s, \sigma)$ we set

$$
A[t, p(s), q(s, \sigma)]=\int_{0}^{t}\left[p(s)+\int_{0}^{s} q(s, \sigma) d \sigma\right] d s,
$$

Received May 16, 2001; revised July 23, 2001. 2000 Mathematics Subject Classification. 26D10, $26 \mathrm{D} 15$.

Key words and phrases. Integral inequalities, discrete analogues, explicit bounds, empty sums and products, hyperbolic partial integrodifferential equation, uniqueness of solutions. 
and for $x, y, s, t, \sigma, \eta \in R_{+}, 0 \leq \sigma \leq s, 0 \leq \eta \leq t$ an some real-valued nonnegative continuous functions $p(s, t), q(s, t, \sigma, \eta)$ we set

$$
B[x, y, p(s, t), q(s, t, \sigma, \eta)]=\int_{0}^{x} \int_{0}^{y}\left[p(s, t)+\int_{0}^{s} \int_{0}^{t} q(s, t, \sigma, \eta) d \eta d \sigma\right] d t d s .
$$

Further, for $n, s, \sigma \in N_{0}, 0 \leq \sigma \leq s$ and some real-valued nonnegative functions $p(s), q(s, \sigma)$ we set

$$
\begin{aligned}
& E[n, p(s), q(s, \sigma)]=\sum_{s=0}^{n-1}\left[p(s)+\sum_{\sigma=0}^{s-1} q(s, \sigma)\right], \\
& \bar{E}[s, p(s), q(s, \sigma)]=p(s)+\sum_{\sigma=0}^{s-1} q(s, \sigma),
\end{aligned}
$$

and for $m, n, s, t, \sigma, \eta \in$ No, $0 \leq \sigma \leq s, 0 \leq \eta \leq t$ and some real-valued nonnegative functions $p(s, t), q(s, t, \sigma, \eta)$ we set

$$
\begin{aligned}
H[m, n, p(s, t), q(s, t, \sigma, \eta)] & =\sum_{s=0}^{m-1} \sum_{t=0}^{n-1}\left[p(s, t)+\sum_{\sigma=0}^{s-1} \sum_{\eta=0}^{t-1} q(s, t, \sigma, \eta)\right] \\
\bar{H}[s, n, p(s, t), q(s, t, \sigma, \eta)] & =\sum_{t=0}^{n-1}\left[p(s, t)+\sum_{\sigma=0}^{s-1} \sum_{\eta=0}^{t-1} q(s, t, \sigma, \eta)\right] .
\end{aligned}
$$

Our main results on integral inequalities are established in the following theorems.

Theorem 1. Let $u(t), a(t), b(t), p(t), q(t, s)$ be nonnegative continuous functions defined for $t, s \in R_{+}, 0 \leq s \leq t$ and $k \geq 0$ be a constant.

$\left(a_{1}\right)$ If

$$
u(t) \leq k+A[t, p(s) u(s), q(s, \sigma) u(\sigma)]
$$

for $t \in R_{+}$, then

$$
u(t) \leq k \exp (A[t, p(s), q(s, \sigma)]),
$$

for $t \in R_{+}$.

$\left(a_{2}\right)$ If

$$
u(t) \leq a(t)+b(t) A[t, p(s) u(s), q(s, \sigma) u(\sigma)]
$$

for $t \in R_{+}$, then

$$
u(t) \leq a(t)+b(t) A[t, p(s) a(s), q(s, \sigma) a(\sigma)] \exp (A[t, p(s) b(s), q(s, \sigma) b(\sigma)]),
$$

for $t \in R_{+}$. 
$\left(a_{3}\right)$ Let $f_{i}: R_{+}^{2} \rightarrow R_{+}, i=1,2$, be continuous functions such that

$$
0 \leq f_{i}(t, u)-f_{i}(t, v) \leq g_{i}(t, v)(u-v),
$$

for $u \geq v \geq 0$, where $g_{i}(t, v)$ are nonnegative continuous functions for $t, v \in R_{+}$. If

$$
u(t) \leq a(t)+b(t) A\left[t, p(s) f_{1}(s, u(s)), q(s, \sigma) f_{2}(\sigma, u(\sigma))\right],
$$

for $t \in R_{+}$, then

$$
\begin{aligned}
u(t) \leq & a(t)+b(t) A\left[t, p(s) f_{1}(s, a(s)), q(s, \sigma) f_{2}(\sigma, a(\sigma))\right] \\
& \times \exp \left(A\left[t, p(s) g_{1}(s, a(s)) b(s), q(s, \sigma) g_{2}(\sigma, a(\sigma)) b(\sigma)\right]\right),
\end{aligned}
$$

for $t \in R_{+}$.

Theorem 2. Let $u(x, y), a(x, y), b(x, y), p(x, y), q(x, y, s, t)$ be nonnegative continuous functions defined for $x, y, s, t \in R_{+}, 0 \leq s \leq x, 0 \leq t \leq y$ and $k \geq 0$ be a constant.

$\left(b_{1}\right)$ If

$$
u(x, y) \leq k+B[x, y, p(s, t) u(s, t), q(s, t, \sigma, \eta) u(\sigma, \eta)],
$$

for $x, y \in R_{+}$, then

$$
u(x, y) \leq k \exp (B[x, y, p(s, t), q(s, t, \sigma, \eta)]),
$$

$\left(b_{2}\right)$ If

for $x, y \in R_{+}$.

$$
\begin{aligned}
& \qquad(x, y) \leq a(x, y)+b(x, y) B[x, y, p(s, t) u(s, t), q(s, t, \sigma, \eta) u(\sigma, \eta)], \\
& \text { for } x, y \in R_{+}, \text {then } \\
& \qquad \begin{aligned}
u(x, y) \leq & a(x, y)+b(x, y) B[x, y, p(s, t) a(s, t), q(s, t, \sigma, \eta) a(\sigma, \eta)] \\
& \times \exp (B[x, y, p(s, t) b(s, t), q(s, t, \sigma, \eta) b(\sigma, \eta)]),
\end{aligned}
\end{aligned}
$$

for $x, y \in R_{+}$.

$\left(b_{3}\right)$ Let $L_{i}: R_{+}^{3} \rightarrow R_{+}, i=1,2$, be continuous functions such that

$$
0 \leq L_{i}(x, y, u)-L_{i}(x, y, v) \leq M_{i}(x, y, v)(u-v),
$$

for $u \geq v \geq 0$, where $M_{i}(x, y, v)$ are nonnegative continuous functions for $x, y, v \in$ $R_{+}$. If

$$
u(x, y) \leq a(x, y)+b(x, y) B\left[x, y, p(s, t) L_{1}(s, t, u(s, t)), q(s, t, \sigma, \eta) L_{2}(\sigma, \eta, u(\sigma, \eta))\right],
$$

for $x, y \in R_{+}$, then

$$
\begin{aligned}
& u(x, y) \leq a(x, y)+b(x, y) B\left[x, y, p(s, t) L_{1}(s, t, a(s, t)), q(s, t, \sigma, \eta) L_{2}(\sigma, \eta, a(\sigma, \eta))\right] \\
& \times \exp \left(B\left[x, y, p(s, t) M_{1}(s, t, a(s, t)) b(s, t), q(s, t, \sigma, \eta) M_{2}(\sigma, \eta, a(\sigma, \eta)) b(\sigma, \eta)\right],\right.
\end{aligned}
$$

for $x, y \in R_{+}$. 
The discrete analogues of Theorems 1 and 2 are given in the following theorems.

Theorem 3. Let $u(n), a(n), b(n), p(n), q(n, s)$ be nonneqative functions for $n, s \in N_{0}$, $0 \leq s \leq n$ and $k \geq 0$ be a constant.

(c) If

$$
u(n) \leq k+E[n, p(s) u(s), q(s, \sigma) u(\sigma)]
$$

for $n \in N_{0}$, then

$$
u(n) \leq k \prod_{s=0}^{n-1}[1+\bar{E}[s, p(s), q(s, \sigma)]]
$$

for $n \in N_{0}$.

(c) If

$$
u(n) \leq a(n)+b(n) E[n, p(s) u(s), q(s, \sigma) u(\sigma)]
$$

for $n \in N_{0}$, then

$$
u(n) \leq a(n)+b(n) E[n, p(s) a(s), q(s, \sigma) a(\sigma)] \prod_{s=0}^{n-1}[1+\bar{E}[s, p(s) b(s), q(s, \sigma) b(\sigma)]]
$$

for $n \in N_{0}$.

(c $\left.c_{3}\right)$ Let $\overline{f_{i}}: N_{0} \times R_{+} \rightarrow R_{+}, i=1,2$, be functions such that

$$
0 \leq \overline{f_{i}}(n, u)-\overline{f_{i}}(n, v) \leq \overline{g_{i}}(n, v)(u-v),
$$

for $u \geq v \geq 0$, where $\overline{g_{i}}(n, v)$ are nonnegative functions for $n \in N_{0}, v \in R_{+}$. If

$$
u(n) \leq a(n)+b(n) E\left[n, p(s) \overline{f_{1}}(s, u(s)), q(s, \sigma) \overline{f_{2}}(\sigma, u(\sigma))\right]
$$

for $n \in N_{0}$, then

$$
\begin{aligned}
u(n) \leq & a(n)+b(n) E\left[n, p(s) \overline{f_{1}}(s, a(s)), q(s, \sigma) \overline{f_{2}}(s, a(\sigma))\right] \\
& \times \prod_{s=0}^{n-1}\left[1+\bar{E}\left[s, p(s) \overline{g_{1}}(s, a(s)) b(s), q(s, \sigma) \overline{g_{2}}(\sigma, a(\sigma)) b(\sigma)\right]\right],
\end{aligned}
$$

for $n \in N_{0}$.

Theorem 4. Let $u(m, n), a(m, n), b(m, n), p(m, n), q(m, n, s, t)$ be nonnegative functions defined for $m, n, s, t \in N_{0}, 0 \leq s \leq m, 0 \leq t \leq n$ and $k \geq 0$ be a constant. 
$\left(d_{1}\right)$ If

$$
u(m, n) \leq k+H[m, n, p(s, t) u(s, t), q(s, t, \sigma, \eta) u(\sigma, \eta)],
$$

for $m, n \in N_{0}$, then

$$
u(m, n) \leq k \prod_{s=0}^{m-1}[1+\bar{H}[s, n, p(s, t), q(s, t, \sigma, \eta)]]
$$

for $m, n \in N_{0}$.

$\left(d_{2}\right)$ If

$$
u(m, n) \leq a(m, n)+b(m, n) H[m, n, p(s, t) u(s, t), q(s, t, \sigma, \eta) u(\sigma, \eta)],
$$

for $m, n \in N_{0}$, then

$$
\begin{aligned}
u(m, n) \leq & a(m, n)+b(m, n) H[m, n, p(s, t) a(s, t), q(s, t, \sigma, \eta) a(\sigma, \eta)] \\
& \times \prod_{s=0}^{m-1}[1+\bar{H}[s, n, p(s, t) b(s, t), q(s, t, \sigma, \eta) b(\sigma, \eta)]]
\end{aligned}
$$

for $m, n \in N_{0}$.

$\left(d_{3}\right)$ Let $\overline{L_{i}}: N_{0}^{2} \times R_{+} \rightarrow R_{+}, i=1,2$, be functions such that

$$
0 \leq \overline{L_{i}}(m, n, u)-\overline{L_{i}}(m, n, v) \leq \overline{M_{i}}(m, n, v)(u-v),
$$

for $u \geq v \geq 0$, where $\overline{M_{i}}(m, n, v)$ are nonnegative functions for $m, n \in N_{0}, v \in R_{+}$. If

$$
u(m, n) \leq a(m, n)+b(m, n) H\left[m, n, p(s, t) \overline{L_{1}}(s, t, u(s, t)), q(s, t, \sigma, \eta) \overline{L_{2}}(\sigma, \eta, u(\sigma, \eta))\right],
$$

for $m, n \in N_{0}$, then

$$
\begin{gathered}
u(m, n) \leq a(m, n)+b(m, n) H\left[m, n, p(s, t) \overline{L_{1}}(s, t, a(s, t)), q(s, t, \sigma, \eta) \overline{L_{2}}(\sigma, \eta, a(\sigma, \eta))\right] \\
\times \prod_{s=0}^{m-1}\left[1+\bar{H}\left[s, n, p(s, t) \overline{M_{1}}(s, t, a(s, t)) b(s, t), q(s, t, \sigma, \eta) \overline{M_{2}}(\sigma, \eta, a(\sigma, \eta)) b(\sigma, \eta)\right]\right],
\end{gathered}
$$

for $m, n \in N_{0}$.

\section{Proofs of Theorems 1-4}

Since the proofs resemble one another, we give the details for Theorems 2 and 3 only. The proofs of Theorems 1 and 4 can be completed by following the proofs of the above mentioned theorems and closely looking at the proofs of the similar results given in [4], see also [5]. 
$\left(b_{1}\right)$ Let $k>0$ and define a function $z(x, y)$ by the right-hand side of (2.8). Then $z(x, y)>$ 0 and nondecreasing for $x, y \in R_{+}, z(x, 0)=z(0, y)=k, u(x, y) \leq z(x, y)$ and

$$
\begin{aligned}
D_{1} z(x, y) & =\int_{0}^{y}\left[p(x, t) u(x, t)+\int_{0}^{x} \int_{0}^{t} q(x, t, \sigma, \eta) u(\sigma, \eta) d \eta d \sigma\right] d t \\
& \leq \int_{0}^{y}\left[p(x, t) z(x, t)+\int_{0}^{x} \int_{0}^{t} q(x, t, \sigma, \eta) z(\sigma, \eta) d \eta d \sigma\right] d t \\
& \leq z(x, y) \int_{0}^{y}\left[p(x, t)+\int_{0}^{x} \int_{0}^{t} q(x, t, \sigma, \eta) d \eta d \sigma\right] d t
\end{aligned}
$$

i.e.

$$
\frac{D_{1} z(x, y)}{z(x, y)} \leq \int_{0}^{y}\left[p(x, t)+\int_{0}^{x} \int_{0}^{t} q(x, t, \sigma, \eta) d \eta d \sigma\right] d t .
$$

Keeping $y$ fixed in (3.1), setting $x=s$ and integrating it with respect to $s$ from 0 to $x$ we get

$$
z(x, y) \leq k \exp (B[x, y, p(s, t), q(s, t, \sigma, \eta)]) .
$$

Using (3.2) in $u(x, y) \leq z(x, y)$ we get (2.9). If $k \geq 0$, we carry out the above procedure with $k+\epsilon$ instead of $k$, where $\epsilon>0$ is an arbitrary small constant, and subsequently pass to the limit as $\epsilon \rightarrow 0$ to obtain (2.9).

$\left(b_{2}\right)$ Define a function $z(x, y)$ by

$$
z(x, y)=B[x, y, p(s, t) u(s, t), q(s, t, \sigma, \eta) u(\sigma, \eta)] .
$$

Then (2.10) can be restated as

$$
u(x, y) \leq a(x, y)+b(x, y) z(x, y) .
$$

From (3.3) and (3.4) we have

$$
\begin{aligned}
z(x, y) & \leq B[x, y, p(s, t)\{a(s, t)+b(s, t) z(s, t)\}, q(s, t, \sigma, \eta)\{a(\sigma, \eta)+b(\sigma, \eta) z(\sigma, \eta)\}] \\
& =B[x, y, p(s, t) a(s, t), q(s, t, \sigma, \eta) a(\sigma, \eta)] \\
& +B[x, y, p(s, t) b(s, t) z(s, t), q(s, t, \sigma, \eta) b(\sigma, \eta) z(\sigma, \eta)] \\
& \leq e(x, y)+B[x, y, p(s, t) b(s, t) z(s, t), q(s, t, \sigma, \eta) b(\sigma, \eta) z(\sigma, \eta)]
\end{aligned}
$$

where $e(x, y)=\epsilon+B[x, y, p(s, t) a(s, t), q(s, t, \sigma, \eta) a(\sigma, \eta)]$, in which $\epsilon>0$ is an arbitrary small constant. Clearly $e(x, y)$ is positive, continuous and nondecreasing in $x, y \in R_{+}$. From (3.5) we observe that

$$
\frac{z(x, y)}{e(x, y)} \leq 1+B\left[x, y, p(s, t) b(s, t) \frac{z(s, t)}{e(s, t)}, q(s, t, \sigma, \eta) b(\sigma, \eta) \frac{z(\sigma, \eta)}{e(\sigma, \eta)}\right] .
$$


Now an application of the inequality in $\left(b_{1}\right)$ to $(3.6)$ yields

$$
z(x, y) \leq e(x, y) \exp (B[x, y, p(s, t) b(s, t), q(s, t, \sigma, \eta) b(\sigma, \eta)]) .
$$

Using (3.7) in (3.4) and letting $\epsilon \rightarrow 0$ we get the required inequality in (2.11).

$\left(b_{3}\right)$ Define a function $w(x, y)$ by

$$
w(x, y)=B\left[x, y, p(s, t) L_{1}(s, t, u(s, t)), q(s, t, \sigma, \eta) L_{2}(\sigma, \eta, u(\sigma, \eta))\right] .
$$

Then (2.13) can be restated as

$$
u(x, y) \leq a(x, y)+b(x, y) w(x, y) .
$$

From (3.8), (3.9) and (2.12) we observe that

$$
\begin{aligned}
w(x, y) \leq & B\left[x, y, p(s, t)\left\{L_{1}(s, t, a(s, t)+b(s, t) w(s, t))-L_{1}(s, t, a(s, t))\right.\right. \\
& \left.+L_{1}(s, t, a(s, t))\right\}, q(s, t, \sigma, \eta)\left\{L_{2}(\sigma, \eta, a(\sigma, \eta)+b(\sigma, \eta) w(\sigma, \eta))\right. \\
& \left.\left.-L_{2}(\sigma, \eta, a(\sigma, \eta))+L_{2}(\sigma, \eta, a(\sigma, \eta))\right\}\right] \\
\leq & E(x, y)+B\left[x, y, p(s, t) M_{1}(s, t, a(s, t)) b(s, t) w(s, t),\right. \\
& \left.q(s, t, \sigma, \eta) M_{2}(\sigma, \eta, a(\sigma, \eta)) b(\sigma, \eta) w(\sigma, \eta)\right],
\end{aligned}
$$

where

$$
E(x, y)=\epsilon+B\left[x, y, p(s, t) L_{1}(s, t, a(s, t)), q(s, t, \sigma, \eta) L_{2}(\sigma, \eta, a(\sigma, \eta))\right],
$$

in which $\epsilon>0$ is an arbitrary small constant. The rest of the proof can be completed by following the last arguments as in the proof of $\left(b_{2}\right)$ given above.

$\left(c_{1}\right)$ Define a function $z(n)$ by the right-hand side of (2.15). Then $z(n)$ is nondecreasing for $n \in N_{0}, z(0)=k, u(n) \leq z(n)$ and

$$
\begin{aligned}
z(n+1)-z(n) & =\left[p(n) u(n)+\sum_{\sigma=0}^{n-1} q(n, \sigma) u(\sigma)\right] \\
& \leq\left[p(n) z(n)+\sum_{\sigma=0}^{n-1} q(n, \sigma) z(\sigma)\right],
\end{aligned}
$$

i.e.

$$
z(n+1) \leq\left[1+p(n)+\sum_{\sigma=0}^{n-1} q(n, \sigma)\right] z(n) .
$$

By setting $n=s$ in (3.11) and then substituting $s=0,1,2, \ldots, n-1$ successively we obtain

$$
\begin{aligned}
z(n) & \leq k \prod_{s=0}^{n-1}\left[1+p(s)+\sum_{\sigma=0}^{s-1} q(s, \sigma)\right] \\
& =k \prod_{s=0}^{n-1}[1+\bar{E}[s, p(s), q(s, \sigma)]] .
\end{aligned}
$$

Using (3.12) in $u(n) \leq z(n)$ we get the desired inequality in (2.16). 
$\left(c_{2}\right)$ Define a function $z(n)$ by

$$
z(n)=E[n, p(s) u(s), q(s, \sigma) u(\sigma)] .
$$

Then $z(0)=0$ and $(2.17)$ can be written as

$$
u(n) \leq a(n)+b(n) z(n) .
$$

From (3.13) and (3.14) we obtain

$$
\begin{aligned}
z(n) & \leq E[n, p(s)\{a(s)+b(s) z(s)\}, q(s, \sigma)\{a(\sigma)+b(\sigma) z(\sigma)\}] \\
& \leq e(n)+E[n, p(s) b(s) z(s), q(s, \sigma) b(\sigma) z(\sigma)],
\end{aligned}
$$

where $e(n)=\epsilon+E[n, p(s) a(s), q(s, \sigma) a(\sigma)]$, in which $\epsilon>0$ is an arbitrary small constant. Clearly, $e(n)$ is positive and nondecreasing for $n \in N_{0}$. From (3.15) we observe that

$$
\frac{z(n)}{e(n)} \leq 1+E\left[n, p(s) b(s) \frac{z(s)}{e(s)}, q(s, \sigma) b(\sigma) \frac{z(s)}{e(s)}\right] .
$$

Now a suitable applications of the inequality in $\left(c_{1}\right)$ to $(3.16)$ yields.

$$
z(n) \leq e(n) \prod_{s=0}^{n-1}[1+\bar{E}[s, p(s) b(s), q(s, \sigma) b(\sigma)]] .
$$

Using (3.17) in (3.14) and letting $\epsilon \rightarrow 0$ we get the required inequality in (2.18).

$\left(c_{3}\right)$ The details of the proof follows by the similar arguments as in the proof of $\left(c_{2}\right)$ and closely looking at the proof of $\left(b_{3}\right)$ given above. Here we omit the details.

\section{Some Applications}

In this section we present applications of the inequality $\left(b_{1}\right)$ in theorem 1 to obtain bound on the solution and uniqueness of solutions of the initial boundary value problem for hyperbolic partial integrodiferential equation of the form

$$
\begin{aligned}
& D_{2} D_{1} z(x, y)=F(x, y, z(x, y))+\int_{0}^{x} \int_{0}^{y} G(x, y, \sigma, \eta, z(\sigma, \eta)) d \eta d \sigma, \\
& z(x, 0)=c_{1}(x), \quad z(0, y)=c_{2}(y), \quad c_{1}(0)=c_{2}(0)=0,
\end{aligned}
$$

where $F: R_{+}^{2} \times R \rightarrow R, G: R_{+}^{4} \times R \rightarrow R, c_{1}, c_{2}: R_{+} \rightarrow R$ are continuous functions.

Our first result gives the bound on the solution of (4.1)-(4.2).

Theorem 5. Suppose that

$$
\begin{aligned}
& |F(x, y, z(x, y))| \leq p(x, y)|z(x, y)| \\
& |G(x, y, \sigma, \eta, z(\sigma, \eta))| \leq q(x, y, \sigma, \eta)|z(\sigma, \eta)|, \\
& \left|c_{1}(x)+c_{2}(y)\right| \leq k
\end{aligned}
$$


where $p, q, k$ are as defined in Theroem 2. If $z(x, y)$ is any solution of (4.1)-(4.2), then

$$
|z(x, y)| \leq k \exp (B[x, y, p(s, t), q(s, t, \sigma, \eta)]),
$$

for $x, y \in R_{+}$.

Proof. The solution $z(x, y)$ of (4.1)-(4.2) satisfies the equivalent integral equation

$$
z(x, y)=c_{1}(x)+c_{2}(y)+\int_{0}^{x} \int_{0}^{y}\left[F(s, t, z(s, t))+\int_{0}^{s} \int_{0}^{t} G(s, t, \sigma, \eta, z(\sigma, \eta)) d \eta d \sigma\right] d t d s .
$$

Using (4.3)-(4.5) in (4.7) we have

$$
|z(x, y)| \leq k+\int_{0}^{x} \int_{0}^{y}\left[p(s, t)|z(s, t)|+\int_{0}^{s} \int_{0}^{t} q(s, t, \sigma, \eta)|z(\sigma, \eta)| d \eta d \sigma\right] d t d s .(4
$$

Now an application of $\left(b_{1}\right)$ in Theorem 2 to (4.8) yields (4.6). The right-hand side of (4.6) gives the bound on the solution $z(x, y)$ of (4.1)-(4.2) in terms of the known functions.

The next result deals with the uniqueness of the solutions of (4.1)-(4.2).

Theorem 6. Suppose that

$$
\begin{aligned}
& |F(x, y, z(x, y))-F(x, y, \bar{z}(x, y))| \leq p(x, y)|z(x, y)-\bar{z}(x, y)|, \\
& |G(x, y, \sigma, \eta, z(\sigma, \eta))-G(x, y, \sigma, \eta, \bar{z}(\sigma, \eta))| \leq q(x, y, \sigma, \eta)|z(\sigma, \eta)-\bar{z}(\sigma, \eta)|,
\end{aligned}
$$

where $p, q$ are as defined in Theorem 2. Then the problem (4.1)-(4.2) has atmost one solution for $x, y \in R_{+}$.

Proof. Let $z(x, y)$ and $\bar{z}(x, y)$ be two solutions of (4.1)-(4.2) for $x, y \in R_{+}$, then we have

$$
\begin{aligned}
z(x, y)-\bar{z}(x, y) & =\int_{0}^{x} \int_{0}^{y}[\{F(s, t, z(s, t))-F(s, t, \bar{z}(s, t))\} \\
& \left.+\int_{0}^{s} \int_{0}^{t}\{G(s, t, \sigma, \eta, z(\sigma, \eta))-G(s, t, \sigma, \eta, \bar{z}(\sigma, \eta))\} d \eta d \sigma\right] d t d s .
\end{aligned}
$$

Using (4.9), (4.10) in (4.11) we have

$$
\begin{aligned}
|z(x, y)-\bar{z}(x, y)| & \leq \int_{0}^{x} \int_{0}^{y}[p(s, t)|z(s, t)-\bar{z}(s, t)| \\
& \left.\left.+\int_{0}^{s} \int_{0}^{t} q(s, t, \sigma, \eta) \mid z(\sigma, \eta)\right)-\bar{z}(\sigma, \eta) \mid d \eta d \sigma\right] d t d s .
\end{aligned}
$$

Now an application of the inequality $\left(b_{1}\right)$ in Theorem 2 with $k=0$ yields $\mid z(x, y)-$ $\bar{z}(x, y) \mid \leq 0$. Therefore $z(x, y)=\bar{z}(x, y)$ for $x, y \in R_{+}$, i.e. there is atmost one solution of the problem (4.1)-(4.2). 
In concluding, we note that the inequalities established in Theorems 2 and 4 can be extended very easily to functions of several independent variables. The precise formulations of these results are very close to that of given above and closely looking at the related results given in $[4,5]$. Here we do not discuss the details. Various applications of other inequalities established here will be given elsewhere.

\section{References}

[1] D. Bainov and P. Simeonov, Integral Inequalities and Applications, Kluwer Academic Publishers, Dordrecht, 1992.

[2] A. A. Martyniuk and R. Gutowski, Integral Inequalities and Stability of Motion, Naukova Dumka, Kiov, 1979(Russian).

[3] D. S. Mitrinović and J. E. Pečarić, Differential and Integral Inequalities, Naucna Knjiga, Belgrade, 1988.

[4] B. G. Pachpatte, Inequalities for Differential and Integral Equations, Academic Press, New York, 1998.

[5] B. G. Pachpatte, Inequalities for Finite Difference Equations, Marcel Dekker Inc., New York, 2002.

[6] B. G. Pachpatte, Some new finite difference inequalities, Comput. Math. Appl. 28(1994), 227-241.

[7] B. G. Pachpatte, On some new inequalities related to a certain inequality arising in the theory of differential equations, J. Math. Anal. Appl. 251(2000), 736-751.

[8] W. Walter, Differential and Integral Inequalities, Springer-Verlag, Berlin, New York, 1970.

57, Shri Niketan Colony, Aurangabad 431 001, (Maharashtra) India. 